\title{
Application of a modified dorsoulnar artery pedicle flap in the repair of thumb tip defects: A case report
}

\author{
TIAN MAO, RENGUO XIE, GUHENG WANG and SHUGUO XING \\ Department of Hand Surgery, Affiliated Hospital of Nantong University, Nantong, Jiangsu 226001, P.R. China
}

Received December 15, 2018; Accepted January 6, 2020

DOI: $10.3892 /$ etm.2020.8583

\begin{abstract}
Between February 2012 and March 201410 patients were admitted to the Affiliated Hospital of Nantong University for surgery due to a thumb tip defect. Nine of these patients were male and one was female and there were 7 cases of left thumb defects and 3 cases of right thumb defects. The surgical procedures followed were first, modification of the flap tail to an equilateral triangle, which facilitated pedicle suturing of soft tissue defects, caused mild tension and effectively reduced venous disorders, and second complete opening of the flap pedicle to the soft tissue defects at the tunnel. All patients were followed up at 6 and 12 months after surgery. Grip and pinch strength were measured 6 and 12 months after surgery. Static two-point discrimination testing of the modified flap showed minor differences from the uninjured hand. Post-surgery grip and pinch strength were restored to approximately $85 \%$ of the level of that in the uninjured hand. The modified dorsoulnar artery pedicle flap provided excellent thumb tip defect coverage and is an effective and safe technique for the restoration of grip and pinch strength to the hand after the repair of a thumb tip defect.
\end{abstract}

\section{Introduction}

The thumb plays a critical role in hand performance and grip. If the thumb is injured overall hand function can be severely affected. Proper repair of thumb soft tissue defects is therefore of significant importance in maintaining hand function (1-3). Soft tissue defects of the thumb are usually accompanied by exposure of the distal phalanx or flexor tendons. Flap surgery is a subspecialty of plastic and reconstructive surgery. In this procedure a piece of tissue is transferred from the donor site to the recipient site while maintaining its own blood supply. These donor tissues may form local flaps (adjacent to the defect site), regional flaps or distant flaps (located at a significant

Correspondence to: Dr Tian Mao, Department of Hand Surgery, Affiliated Hospital of Nantong University, 20 Xisi Road, Nantong, Jiangsu 226001, P.R. China

E-mail: tianmao17@yeah.net

Key words: triangular flap, grip strength, pinch strength distance from the donor site) (4). Multiple techniques and methods have been employed to repair soft tissue defects of the thumb tip (5). Mitsunaga et al (6) demonstrated that a modified digital artery perforator flap allowed for preservation of digital length, volume and finger function. These flaps can be raised as adiposal-only flaps or extended flaps and supercharged through perforator-to-perforator anastomoses. For traumatic fingertip and finger stump reconstructions, digital artery perforator flaps deliver consistent aesthetic and functional results (7-9). Subsequently, numerous clinical trials have demonstrated that the thumb dorsoulnar artery pedicle flap is an effective technique to repair soft tissue defects (7-14). Ramirez and Gonzalez (15) described the local patterns of the thumb vascular anatomy of 30 fresh right hands from male and female cadavers using a vascular injection technique with methyl methacrylate, providing anatomical evidence for the pedicle flap design. However, conventional flap design has certain limitations, which reduce the clinical efficacy of soft tissue repair, due to the tension in the subcutaneous tunnel, and lower the survival rate of the flap. In the present case report, thumb dorsoulnar artery pedicle flaps tunneled during flap transposition were designed to repair thumb tip defects and their success was evaluated through tests of hand grip and pinch strength.

\section{Patients and methods}

Patient information and study design. Between February 2012 and March 2014, 10 modified dorsoulnar artery pedicle flaps were utilized in 10 patients ( 9 males and 1 female). The area of the thumb tip defects ranged from 2 to $9 \mathrm{~cm}^{2}$ in size (Fig. 1). These tip defects were caused by crush, cutting, avulsion or wringer injuries. The mean patient age was 47 years, with a range from 24 to 60 years. Seven cases involved left thumb injury and three involved right thumb injury. The phalanx or tendon was exposed in all cases.

The study inclusion criteria were as follows: i) The soft tissue defect was on the palm side of the hand; ii) the end point of the flexor pollicis longus tendon was not damaged; and iii) there was no obvious damage to the nail bed. The study exclusion criteria were: i) The patient had defects in the flexor pollicis longus tendon; ii) the patient had suffered damage to more than $1 / 4$ of the nail bed; iii) the proximal end of the wound was beyond the interphalangeal joint; and iv) surgery could not be tolerated due to systemic disease. 
Emergency debridement was delivered before surgical procedures were performed using thumb dorsoulnar artery pedicle flaps. All patients underwent surgery between 1 and 4 days after injury. All cases were surgically treated with modified dorsoulnar artery pedicle flaps (Table I).

Triangular flap design. All cases were subject to brachial plexus anesthesia. All necrotic and poorly vascularized tissues were thoroughly debrided and utilized as the recipient site of modified flaps. The size of the tip defects was measured and then the donor site of the modified flaps was marked as follows: i) The connection between the snuffbox proximal and the metacarpophalangeal joints of the first dorsal ulnar side was marked as the central line of the flap. To ensure adequate vascularity, the donor site flap was at least $0.5 \mathrm{~cm}$ away from the proximal interphalangeal joint of the thumb; ii) the flap tail was modified to an equilateral triangle (Fig. 2); iii) the coverage of the donor flap was slightly greater than that of the defect, or more specifically the external portion of the turnover flap was slightly oversized; and iv) the distal pivot point was $>0.7-1 \mathrm{~cm}$ from the tips of the defects (the distal end of flap was configured as an equilateral triangle to fully cover the pedicle when rotating the flap).

Written informed consent was obtained from all patients. The study procedures were approved by the Ethics committee of the Affiliated Hospital of Nantong University.

Flap transfer. All flap transfers were performed by an experienced hand surgeon (Dr Renguo Xie) accredited with expertise level 4 for flap surgery (16-18). The skin at the distal end was excised and the thumb dorsal artery was exposed. An incision was made from the edge to the proximal end of the flap and the thumb dorsal ulnar artery was ligated. From the thumb extensor aponeurosis and superficial adductor muscle membrane, the flap containing a portion of the sarcolemma was turned over in the opposite direction. The flap, $1.0 \mathrm{~cm}$ in length and $0.8 \mathrm{~cm}$ in width, containing the thumb dorsal ulnar artery and 1 to 2 dorsal veins was designed along the dorsal ulnar artery. The flap pedicle was located at either ulnar side. The proximal part of the flap was used as the pedicle. The point of rotation did not exceed one half of the plane of the middle phalanx (Fig. 2). After turning over the flap, to cover the wound area, the flap was sutured without any tension. The superficial branch of the radial nerve was fixed to coaptate to the digital nerve, to recover the nerve sensation. It could be directly sutured due to the small donor site (Fig. 3). If turning over the flap led to tension, then a full-thickness skin graft from the inner aspect of the forearm could be used. The blood vessel distribution, color/temperature change and flap survival rate of the flap were closely observed within $24 \mathrm{~h}$ after surgery. The suture was removed 14 days after surgery.

Strength measurements. Measuring grip and pinch strength is an important part of hand injury evaluation. Grip strength was measured with a Jamar hand dynamometer and the pinch strengths were measured with a Jamar hydraulic pinch gauge (Patterson Medical Products, Inc.), the non-injured hand was used as the control. The elbow was bent at $90^{\circ}$, the forearm was in a neutral position, the wrist was flexed from $0^{\circ}$ to $30^{\circ}$, and the scale deviation was $0^{\circ}$ to $15^{\circ}$. Each test was repeated
3 times at 2 min intervals and all evaluations were performed by a single investigator.

Statistical analysis. Grip and pinch strength were analyzed with a paired t-test using the SPSS version 18.0 (SPSS, Inc.). Data were analyzed in triplicate and were presented as the mean and standard deviation. $\mathrm{P}<0.05$ was considered to indicate a statistically significant difference.

\section{Results}

Postoperative flap survival. All the modified flaps in the 10 patients survived. The flaps survived at the skin graft donor sites in four patients and after direct suture in the remaining 6 cases. Eight patients obtained complete survival of the modified flaps, whereas two patients suffered from distal flap necrosis. Subsequently, the necrotic tissues were removed and replaced with healthy tissue at $\sim 5$ weeks after the first procedure. One patient presented with postoperative venous insufficiency, which was effectively managed by the treatment described below.

Postoperative thumb motion and function. Postoperatively, 10 patients underwent follow-up for 6 to 12 months. The modified flaps were observed to be in good condition (Fig. 4). Two measurements were taken at 6 and 12 months after surgery. The range of motion of the thumb interphalangeal joint ranged from $5^{\circ}$ to $75^{\circ}$, and for the metacarpopalangeal joint $5^{\circ}$ to $65^{\circ}$, suggesting that partial recovery of static two-point discrimination had been achieved in the thumb flaps (Fig. 5). For postoperative coverage of the donor sites, skin grafts were utilized in 4 cases, and the donor sites were directly sutured in the remaining 6 patients. One case exhibited venous insufficiency, which was resolved by therapy and one case lost the distal flexor pollicis longus tendon. First, the pedicle suture of the flap was removed so that the pedicle was relaxed and did not squeeze the vein. One or two veins were identified at the distal edge of the flap. The dark venous blood at the edge of the flap was released by the surgeon until bright red arterial blood appeared and these procedures were repeated every $30 \mathrm{~min}$ by family members. The venous insufficiency was monitored hourly by surgeons and had disappeared within $12 \mathrm{~h}$.

Grip and pinch strength were measured twice, 6 and 12 months after using a modified dorsoulnar artery pedicle flap in the repair of the thumb tip defects. None of the patients started new medication. Grip measurement was related to the second position of the dynamometer and was performed with the elbow at $\sim 90^{\circ}$ according to the American Society of Hand Therapists recommendations (19). Pinch strength was measured between the tip of the thumb and index finger. Three consecutive measurements were performed with a $2 \mathrm{~min}$ inter-measurement interval. At 6 and 12 months after surgery, the grip and pinch strength of the affected hands were restored to $85 \%$ of the normal side ( $P>0.05$; Table II).

The flap sutures and the flap pedicle were released, the hematocele inside the tunnel removed, the flap pedicle covered with vaseline gauze, and the tunnel skin sutured at postoperative day 7. Mild adverse events occurred throughout the surgical procedures, which were handled in a timely manner. 
Table I. Baseline data of 10 patients included in the current study.

\begin{tabular}{lcclccc}
\hline Sex & Age & Injury side & Cause of injury & Postoperative necrosis & Flap size $(\mathrm{cm})$ & Survival condition \\
\hline Male & 43 & Right & Machine & No & $3 \times 2.5$ & Healed \\
Female & 57 & Left & Machine & No & $4 \times 3$ & Healed \\
Male & 40 & Left & Machine & Partial & $3.5 \times 2$ & Healed \\
Male & 24 & Left & Machine & No & $4 \times 2.5$ & Healed \\
Male & 59 & Left & Machine & No & $3 \times 2$ & Healed \\
Male & 40 & Right & Machine & No & $3 \times 2$ & Healed \\
Male & 60 & Left & Machine & No & $2 \times 1$ & Healed \\
Male & 56 & Left & Crush injury & Partial & $2 \times 1$ & Healed \\
Male & 48 & Right & Machine & No & $2 \times 1.6$ & Healed \\
Male & 43 & Left & Crush injury & No & $2.3 \times 1.4$ & Healed \\
\hline
\end{tabular}

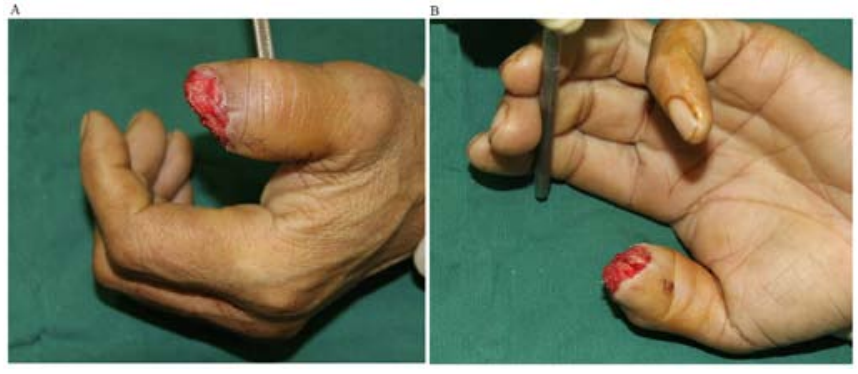

Figure 1. Thumb soft tissue defect. An (A) Dorsal and (B) ventral view of the thumb soft tissue defect.

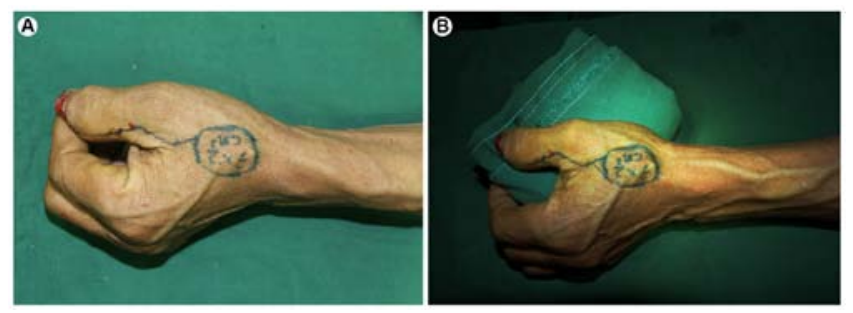

Figure 2. Design of the modified dorsoulnar artery pedicle flap. (A) Hand dorsal view and (B) anatomical structure of the flap.

\section{Discussion}

Overall, the modified dorsoulnar artery pedicle flap is an efficacious and safe technique for the repairment of the thumb tip defects in clinical settings. In this case report, the range of motion of interphalangeal joint activity was $5-75^{\circ}$ and $5-45^{\circ}$ for the metacarpophalangeal joint activity. Though the range of motion improved, it did not recover completely. This may be due to several potential factors. It is possible that scar tissue extended into the metacarpophalangeal joints when the incision was healed, as the incision was created from the snuffbox to the thumb wound through the ulnar metacarpophalangeal joint. Another possibility is that a majority of patients resisted performing joint movement during the early stages of recovery, due to poor tolerance of the severe pain. It is also possible that the, partial tumescent flaps impacted interphalangeal joint flexion. In one case, the distal flexor pollicis longus tendon was lost.
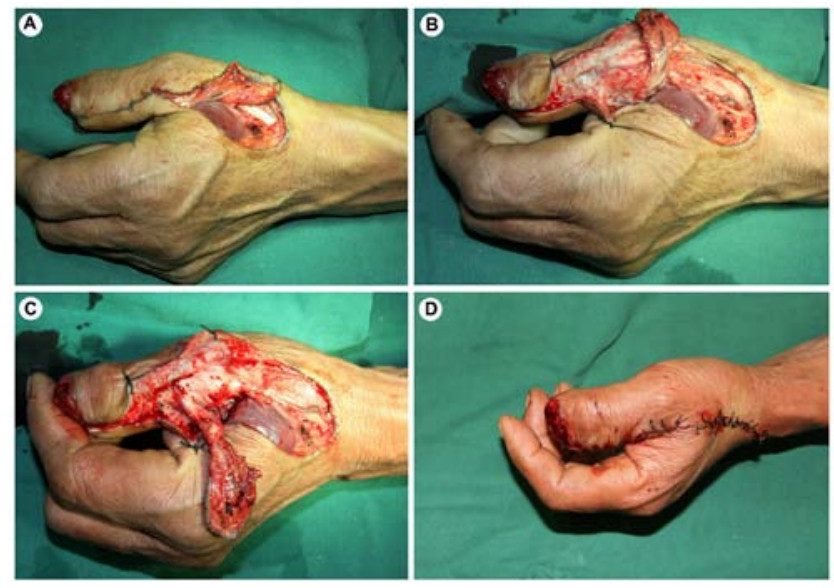

Figure 3. Incision and suture of the modified flap. (A) Anatomical view of the initial flap.(B) Anatomical view of the first metacarpal dorsal site. (C) Anatomical view of the thumb artery and (D) postoperative view of the flap suture.
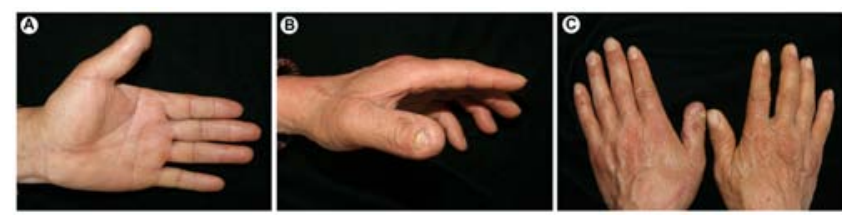

Figure 4. The shape and contour of the flap at 6 months postoperative. (A) View of the palm. (B) Lateral view. (C) Comparison between bilateral hands.

The present case study reports the design of a distal flap through the creation of a dorsoulnar flap with an equilateral triangle tail. Concurrently, the thumb wound was directly coapted with the pedicle and the flap embedded in the tip of the shuttle-type tunnel, which could avoid the incidence of pedicle entrapment and reduce the risk of flap necrosis. Both the donor and recipient sites functioned properly and the donor site could be directly sutured. No apparent difference was identified in the grafted skin color between the donor and recipient sites. The risk of severe damage to the major vascular supply of the thumb could be averted. The modified thumb flaps were suitable for large degrees of rotation in a large area. A long vascular pedicle with a flexible transfer could be created, which could conveniently reach the tip of the wound site. The 
Table II. Grip and pinch strengths at 6 and 12 months after surgery.

\begin{tabular}{|c|c|c|c|c|c|c|}
\hline & \multicolumn{2}{|c|}{6 months } & \multirow[b]{2}{*}{ P-value } & \multicolumn{2}{|c|}{12 months } & \multirow[b]{2}{*}{ P-value } \\
\hline & Affected hand & Normal hand & & Affected hand & Normal hand & \\
\hline Pinch Strength (kg) & $3.62 \pm 1.77$ & $3.82 \pm 1.80$ & 0.175 & $3.73 \pm 1.85$ & $3.82 \pm 1.80$ & 0.137 \\
\hline Grip strength (kg) & $27.3 \pm 8.3$ & $32.0 \pm 11.4$ & 0.186 & $28.4 \pm 7.12$ & $32.0 \pm 11.4$ & 0.143 \\
\hline
\end{tabular}
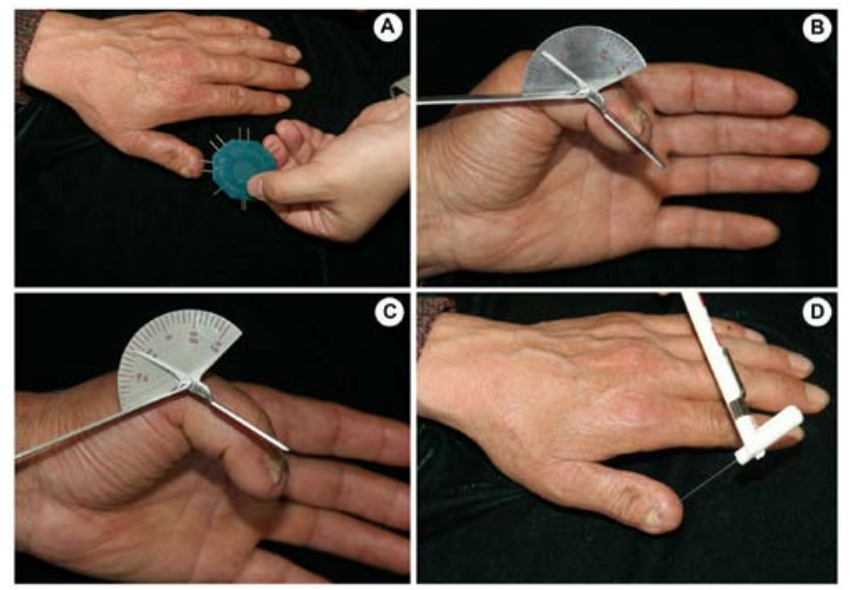

Figure 5. Multiple tests of the modified flap at postoperative 6 months (A) Two-point discrimination test (28). (B) Range of motion test between fingers (29). (C) Range of motion test of the finger joint (29). (D) Nerve sensation test (30).

follow-up data at 6 and 12 months post-surgery showed that grip and pinch strength of the affected hands was restored to $85 \%$ of the normal side.

Bertehlli and Koury (5) improved the configuration of thumb dorsal ulnar artery pedicle flaps and prolonged flap survival in 30 patients, using a technique termed the Brunelli flap. Yu et al (9) evaluated the functional sensory recovery of random-pattern abdominal skin flaps in the repair of fingertip cutaneous deficiency and found that all flaps obtained satisfactory flexibility and texture and that sensory recovery was achieved. Hrabowski et al (20) reported that the maximum area of the harvested thumb radial dorsal artery island flap was $5 \times 4 \mathrm{~cm}^{2}$, slightly larger than $4 \times 3 \mathrm{~cm}^{2}$ in the present case study. Moschella and Cordova (21) demonstrated that the large area of radial flap, which could be harvested, might readily lead to venous congestion, even necrosis. In a previous study, it was determined that the skin of the donor site lacks flexibility after direct suture and this affected the perception of patients' towards the results. Traditionally, the thumb dorsal ulnar artery flap was a round or oval shape. The pedicle could reach the thumb defects through a closed tunnel.

Wang et al (22) reported that the furthest communicating branch between the thumb palmar artery and thumb dorsal artery was located approximately $0.5 \mathrm{~cm}$ from the proximal interphalangeal joint. The thumb dorsal artery island flap, which contained superficial radial nerve branches, was harvested from the first metacarpal dorsal site. It would be prudent to restore sensory function when suturing the superficial radial nerve and the digital nerve (22). In previous studies, the flap nerve was directly sutured with the digital nerve stump, whereas the result was not satisfactory, especially for two-point discrimination test (9-14). Similar findings were obtained in the present case report, though the thumb flap two-point discrimination was partially restored.

Local flaps can be further divided according to their blood supply. Random pattern flaps have no known feeding blood vessel, while axial pattern flaps are based on a known artery that directly supplies a specific skin territory. Interconnections between branches of adjacent axial vessels exist that connect neighboring skin territories. The V-Y advancement flap is suitable for the coverage of transverse or dorsal oblique fingertip amputations with exposed bone and sufficient nail bed support and length (23). The thenar flap is indicated for volar skin avulsions over the pulp of the finger (24). The cross-finger flap is more suited to treat distal defects, in which more tissue is required for coverage than can be obtained from a local advancement flap such as V-Y flap (25). The homodigital island flap that is based on the volar blood supply of the fingers, either the radial or ulnar digital artery and its venae comitantes (26). The dorsal metacarpal artery flap is based on a constant palmar-dorsal perforator present in the digital web-space and it increases the span of the flap to reach more distal defects (27).

In the present case report, the two-point discrimination of the thumb flaps was slightly different than that of the healthy side. Grip and pinch strength of the affected hands could be restored to $85 \%$ of the normal side. The only partial recovery of two-point discrimination was likely associated with the adverse events induced by soft tissue injury. For the eight patients undergoing postoperative follow-up, two-point discrimination of the flaps was inferior to that of the healthy side, but it did not affect the motion of the hands. Hand motion was fully restored at postoperative 12 months, whereas nerve sensation was not fully recovered, probably because the modified thumb flaps do not carry complete innervation. The length of the thumb was almost retained, the wound was fully healed and the function of the thumb was slightly impaired. Taken together, the grip and pinch strength of the affected hands were slightly less than that of the contralateral side. During postoperative follow-up, partial flap necrosis occurred in 2 patients. The dorsal ulnar artery was injured during surgical exploration, which led to constrained flap vascularization and subsequent flap necrosis. In one case, the venous return of the flap became unobstructed after treatment

Triangular flaps were used for the repair of 10 thumbs, showing that this technique not only resolved the tension of the subcutaneous tunnel and, but also improved the survival rate of the flap when compared to previous experience. The advantage of this method is that the flap blood vessels are stable; the amputation surface can be completely covered. However, this 
method cannot be used for skin defects of a finger supplied by a dorsoulnar artery, which limits its application in hand surgery.

In conclusion, application of triangular dorsoulnar artery pedicle flap in repair of thumb tip defects improved the tension of the subcutaneous tunnel and the flap survival rate and restored the grip and the pinch strength for the repaired hand. At 6 and 12 months after the modified dorsoulnar artery pedicle flap had been used to repair the thumb tip defects, the grip and pinch strength of the affected hands had been restored to $85 \%$ of that in the normal side. The modified dorsoulnar artery pedicle flap is an efficient and safe technique to repair thumb tip defects.

\section{Acknowledgements}

The authors would like to thank Ms. Lihua Chen (Operating Room Nursing Department of the Affiliated Hospital of Nantong University) for her surgical assistance.

\section{Funding}

No funding was received.

\section{Availability of data and materials}

The datasets used and/or analyzed during the present study are available from the corresponding author on reasonable request.

\section{Authors' contribution}

TM proposed and designed this modified skin flap, performed all surgeries, performed research and completed the manuscript. RX participated in the design of the skin flap and part of the surgery. GW and SX participated in part of the surgery and collected and analyzed the data. All authors read and approved the final manuscript.

\section{Ethics approval and consent to participate}

Written informed consent was obtained from all patients. The study procedures were approved by the Ethics committee of the Affiliated Hospital of Nantong University.

\section{Patient consent for publication}

Patients provided written consent for the publication of their data.

\section{Competing interests}

The authors declare that they have no competing interests.

\section{References}

1. Muzaffar AR, Chao JJ and Erdidrich JB: Posttraumatic thumb reconstruction. Plast Rdconstr Surg 116: 103e-122e, 2005.

2. Vdder NB and Hanel DP: The mangled upper exteremity. In: Green's Operative Hand Surgery. Green D (ed). 5th edition. Elsevier/Churchill Livingstone, New York, NY, pp1587-1628, 2005.

3. Tang JB, Elliot D, Adani R, Saint-Cyr M and Stang F: Repair and reconstruction of thumb and finger tip injures: A global view. Clin Plast Surg 41: 325-359, 2014.
4. Foucher G, Boulas HJ and Braga Da Silva J: The use of flaps in the treatment of fingertip injuries. World J Surg 15: 458-462, 1991.

5. Bertehlli JA and Koury Z: Neurocutaneous island flap in the hand: Anatomical basis and preliminary results. Br J Plast Surg 45: 586-590, 1992.

6. Mitsunaga N, Mihara M, Koshima I, Gonda K, Takuya I, Kato H, Araki J, Yamamoto Y, Yuhei O, Todokoro T, et al: Digital artery perforator (DAP) flaps: Modifications for fingertip and finger stump reconstruction. J Plast Reconstr Aesthet Surg 63: 1312-1317, 2010.

7. Zhu L, Xu Q, Kou W, Ning B and Jia T: Outcome of free digital artery perforator flap transfer for reconstruction of fingertip defects. Indian J Orthop 48: 594-598, 2014

8. Usami S, Kawahara S, Yamaguchi Y and Hirase T: Homodigital artery flap reconstruction for fingertip amputation: A comparative study of the oblique triangular neurovascular advancement flap and the reverse digital artery island flap. J Hand Surg Eur Vol 40: 291-297, 2015

9. Yu YD, Zhang YZ, Bi WD and Wu T: Functional sensory function recovery of random-pattern abdominal skin flap in the repair of fingertip skin defects. Exp Ther Med 5: 830-834, 2013.

10. Panse $N$ and Sahasrabudhe P: The ulnar digital artery perforator flap: A new flap for little finger reconstruction-a preliminary report. Indian J Plast Surg 43: 190-194, 2010.

11. Horta R, Barbosa R, Oliveira L, Amarante JM, Marques M, Cruz Reis J and Rebelo M: Neurosensible reconstruction of the thumb in an emergency situation: Review of 107 case. Tech Hang Up Extrem Surg 13: 85-89, 2009.

12. Teran P, Carnero S, Miranda R, Trillo E and Estefanía M: Refinements in dorsoulnar flap of the thumb: 15 cases. J Hand Surg Am 35: 1356-1359, 2010.

13. Cheah AE and Chong AK: Soft-tissue coverage of the hand. Curr Orth Practice 23: 336-345, 2012.

14. Han D, Sun H, Jin Y, Wei J and Li Q: A technique for the non-microsurgical reconstruction of thumb tip amputations. J Plast Reconstr Aesthet Surg 66: 973-977, 2013.

15. Ramirez AR and Gonzalez SM: Arteries of the thumb: Description of anatomical variations and review of the literature. Plast Reconstr Surg 129: 468e-476e, 2012.

16. Tang JB: Uncommon methods of flexor tendon and tendon-bone repairs and grafting. Hand Clin 29: 215-221, 2013.

17. Tang JB: Re: Levels of experience of surgeons in clinical studies. J Hand Surg Eur Vol 34: 137-138, 2009.

18. Tang JB and Avanessian B: Clinical reports: The importance of reporting a surgeon's level of expertise. J Hand Surg Am 40: 416-417, 2015.

19. Fess EE and Moran C: Clinical Assessment Recommendations. American Society of Hand Therapists, Indianapolis, 1981.

20. Hrabowski M, Kloeters O and Germann G: Reverse homodigital dorsoradial flap for thumb soft tissue reconstruction: Surgical technique. J Hand Surg Am 35: 659-662, 2010.

21. Moschella F and Cordova A: Reverse homodigital dorsal radial flap of the thumb. Plast Reconstr Surg 117: 920-926, 2006.

22. Wang $\mathrm{C}, \mathrm{Xu} \mathrm{J}$ and Zhang L: Applied anatomy of the island flap pediceled with dorsal digital vessels. Chin J Clin Anat 24: 514-517, 2006.

23. Ramirez MA and Means KR Jr: Digital soft tissue trauma: A concise primer of soft tissue reconstruction of traumatic hand injuries. Iowa Orthop J 31: 110-20, 2011.

24. Gatewood A: A plastic repair of finger defects without hospitalization. JAMA 87: 1479, 1926.

25. Cronin TD: The cross finger flap: A new method of repair. Am Surg 17: 419-425, 1951.

26. Weeks PM and Wray RC (eds): Management of Acute Hand Injury: A Biological Approach. Mosby, St. Louis, MI, 1973.

27. Maruyama Y: The reverse dorsal metacarpal flap. Br J Plast Surg 43: 24-27, 1990.

28. Dellon AL: Management of peripheral nerve problems in the upper and lower extremity using quantitative sensory testing. Hand Clin 15: 697-715, 1999.

29. Nizamis K, Rijken NH, Mendes A, Janssen MM, Bergsma A and Koopman BF: A novel setup and protocol to measure the range of motion of the wrist and the hand. Sensors (Basel) 18: E1881, 2018.

30. MacDermid JC: Measurement of health outcomes following tendon and nerve repair. J Hand Ther 18: 297-312, 2005.

This work is licensed under a Creative Commons Attribution-NonCommercial-NoDerivatives 4.0 International (CC BY-NC-ND 4.0) License. 